\title{
The medical history as a diagnostic technology
}

Nick Summerton

\begin{abstract}
The medical history is a powerful diagnostic technology. However, in seeking to establish an appropriate balance between the history and the other diagnostic modalities more explicit consideration must be given to the performance characteristics of the medical history. Building on recent work undertaken in the UK and elsewhere in Europe it is now feasible to develop a library of setting-specific likelihood ratios and $\mathrm{\kappa}$ statistics for key elements of the medical history. Of particular importance to those working in primary care, statistically adjusted combinations of information from the medical history can be generated; furnishing clinicians with likelihood ratios of significant magnitudes. It is suggested that developing a more rational approach to the use of the medical history could lead to improvements in diagnostic efficiency and effectiveness, with benefits for individual patient care in addition to the overall NHS budget. When diagnosis is viewed as a processing pathway founded on a robust medical history, it becomes clear than in some situations investigations may become unnecessary and, in other circumstances, their impact will be enhanced.

Keywords

diagnosis; Bayes theorum; likelihood ratios; primary care.
\end{abstract}

\section{INTRODUCTION}

Has the medical history become redundant? Over 30 years ago Hampton and colleagues suggested that the history determined $83 \%$ of the diagnoses in medical outpatients. ${ }^{1}$ However, with the rapid growth in new diagnostic technologies there is now a suggestion that it is more efficient and cost-effective to employ a technician to undertake a battery of investigations rather than have an expensive clinician spending time listening to patients. In his recent speech on the health service the Prime Minister, Gordon Brown, stated 'utilising these new technologies must be at the heart of any progressive health policy'.2

Diagnostics is a central element within the Department of Health's initiative, designed to deliver an 18-week patient pathway from GP referral to the start of treatment. However, whereas there is a particular focus on endoscopy, imaging, pathology, and physiology testing, with the appointment of four clinical leads in these areas, the medical history has not been afforded any similar special prominence. ${ }^{3}$ Furthermore, although the National Institute for Health and Clinical Excellence (NICE) is charged with appraising diagnostic technologies, its remit is restricted to those newer technologies that are CE marked. ${ }^{4}$ Nowadays it also seems as if anyone is permitted to take a medical history without the requirement for any specific training to understand the complexities of symptom reporting, symptom evolution, symptom classification, or symptom interpretation. ${ }^{5,6}$

This trend towards investigation-centred diagnosis is misplaced. Aside from the direct physical risks associated with some of the more invasive investigations there are major cost implications to consider. For example, in a Department of Health press release highlighting the expansion in magnetic resonance imaging (MRI) scanning, the accompanying notes stated that 'MRI scans help diagnose ... acute or chronic migraine and headaches'. ' However, using Bayes' theorem it can be calculated that in those with a new onset unilateral headache and nausea presenting to a primary care clinician the probability of migraine after taking a medical history is already greater than $80 \%\left(\right.$ Box 1). ${ }^{8,9}$ 


\section{Box 1. Calculating post-history probability using Bayes' theorem.}

Formula: Odds ratio form of Bayes' theorem:

posterior odds $=$ likelihood ratio $(\mathrm{LR}) \times$ prior odds

- Published data: Prior probability of migraine in patients presenting to primary care clinicians with new-onset headache: $5.3 \%{ }^{8}$

Positive LRs for specific additional symptoms/symptom features in patients presenting with new onset headaches: unilateral headache $(L R=3.1)$; nausea $(L R=23.2){ }^{9}$

- Computation: Post-history odds = positive LR (for unilateral headache, that is, 3.1) $x$ positive LR (for nausea, that is, 23.2) $x$ prior odds

- Convert the prior probability (5.3\%) to odds using odds = probability/1-probability (that is, odds $=0.056$ )

- Multiple the odds by the LR product (that is, $0.056 \times 71.9$ ) $=4.03$

- Convert back to probabilities using probability = odds $/ 1+$ odds (that is, $4.03 / 5.03$ ) $=80 \%$

NB: In this simple calculation I have assumed that the positive LRs are independent of each other (referred to as the independence or naive Bayes' approach).

An overarching focus on investigations also highlights a failure to appreciate that not every 'abnormality' is synonymous with an organic disease: no specific physical disorder can be established as the cause in between $30 \%$ and $75 \%$ of patients with symptoms. ${ }^{10}$

In common with any diagnostic tool the medical history certainly has its deficiencies. However, unlike the other diagnostic modalities a continuing absence of any specific focus on the medical history by senior clinicians or policymakers has resulted in some fundamental issues remaining unaddressed. For example, we persist in questioning patients about symptoms that exhibit only moderate reliability (Box $2)$, such as 'change in bowel habit' $(\kappa=0.51) .{ }^{11}$ Although reported rectal bleeding might be more reliable $(\kappa=0.85)$ we may fail to check that the response is valid: how many of us ask the patient whether they actually look at their motions or consider if they could spot a small amount of blood within the toilet pan of a dimly lit cloakroom?

A number of measures such as sensitivity, specificity, likelihood ratio, and predictive value have been used to determine the discriminant ability of the medical history. The positive predictive value (that is,

\section{Box 2. Reliability.}

Reliability (or reproducibility) is the extent to which repeated measurements of a stable phenomenon produce similar results. To assess the chance-corrected proportional agreement the $\kappa$ statistic is used and different ranges for $\kappa$ have been categorised with respect to the degree of agreement they suggest. Values greater than 0.80 may be taken to represent almost perfect agreement, values between 0.61 and 0.80 substantial agreement, values between 0.41 and 0.60 moderate agreement, values between 0.21 and 0.40 slight agreement, and values of 0.20 or below represent poor agreement beyond chance. ${ }^{12}$ the probability that the disease is present if the patient has, for example, a specific symptom) often makes the most intuitive sense to clinicians, and yet is a constant source of misunderstanding between GPs and our secondary colleagues. It is imperative to be aware that the predictive value is affected by prevalence: as the prevalence falls, the number of false positives tends to increase, resulting in a lowering of the positive predictive value. The effect of prevalence can also be readily understood in relation to the odds ratio version of Bayes' theorem referred to earlier (see Box 3 for detailed definitions).

Interestingly, Sox and colleagues have demonstrated that the posterior odds for coronary artery disease in two primary care populations was lower than in a referred arteriography population, even when patients with virtually identical clinical histories were compared. ${ }^{13}$

Unfortunately, for the primary care clinician the prevalence problem (and the reduced magnitude of the prior odds) is compounded by the finding that many items in the medical history, such as presenting (iatrotropic) or elicited (non-iatrotropic) symptoms, have positive likelihood ratios that are too small to be clinically useful. A patient attending with dysuria might have a urinary tract infection but the positive likelihood ratio provided by this particular presenting symptom only amounts to $1.5 .{ }^{14}$ In relation to the diagnosis of pneumonia in a patient with a cough, eliciting a history of sputum production merely affords an additional positive likelihood ratio of $1.3 .^{15}$ For a number of inexperienced or younger doctors this may perhaps account for some of their tendency to order an increasing number of diagnostic tests. ${ }^{16}$

Considering the iatrotropic symptom (for example, abdominal pain or headache) in conjunction with other information from the medical history (for example, noniatrotropic symptoms, patient demographics, current and past treatments) to enhance the magnitude of the collective positive likelihood ratio represents an

\section{How this fits in}

In seeking to establish an appropriate balance

between the medical history and the other

diagnostic modalities more explicit consideration

must be given to the performance characteristics of

the history in the context of a diagnostic

processing pathway. It is now feasible to develop a

library of setting-specific likelihood ratios and $\kappa$

values for individual features and combinations of

items of medical history information. 
alternative approach. For the non-specific headache of temporal arteritis it seems that a temporal location only produces a positive likelihood ratio of 1.5 and most GPs would simply move on to request an erythrocyte sedimentation rate (ESR). However, although an ESR between 61 and 80 confers an additional positive likelihood ratio of 1.4, establishing a clear history of jaw claudication provides an additional positive likelihood ratio of 4.2 , and diplopia an additional positive likelihood ratio of 3.4. ${ }^{17}$ However, it is important to note that the negative likelihood ratios of these features are only 0.7 and 0.9 respectively, indicating that their absence is much less helpful in arguing against the diagnosis of temporal arteritis.

Medical history clusters represent a more robust approach to linking history data together. For example, Muris and colleagues have demonstrated that within the primary care setting, a combination of age greater than 65 years, male sex, non-specific abdominal pain, and unexplained weight loss provides a posterior probability for neoplastic disease of $50 \% .{ }^{18}$ Moreover, it is now feasible to generate a large number of such clusters using data from recent work undertaken in the Netherlands, Belgium, the UK, and Scandinavia. However, careful consideration needs to be given to the potential lack of independence between individual items of clinical information; the net discriminatory value of the combinations often being slightly lower than predicted from simply multiplying the likelihood ratios together. For example, in relation to the diagnosis of urological cancer in patients with new onset haematuria it seems that the unadjusted combination of male sex, hesitancy, poor stream, and having at least three episodes of haematuria would overestimate the combined positive likelihood ratio by $15 \% .{ }^{19} \mathrm{~A}$ number of statistical approaches have now been developed based on independence Bayes' theorem or logistic regression to facilitate such adjustments. In a recent review it was suggested that Albert's method, which includes an offset term to estimate adjusted likelihood ratios for combinations of clinical information, is the most straightforward approach. ${ }^{20}$

A patient who comes to see their primary care physician about a cough will already have a prior (existing) probability of pneumonia. This probability will be modified by additional information derived from the medical history to arrive at a new (post-history) probability of pneumonia. This probability may, in turn, be further adjusted by data derived from the clinical examination to produce a post-examination probability that, after a chest radiograph, could then become a post-test probability. Thus, in many situations diagnosis is best viewed as a diagnostic processing pathway incorporating a number of

\section{Box 3. Bayesian terminology.}

Bayes' theorem (odds ratio form): A theorem which permits the calculation of the posterior odds of a disease by knowing the prior odds of the disease and the likelihood ratio (LR) of the clinical information being used. That is, posterior odds = prior odds $\times$ LR

Likelihood ratio: The ratio of the probability of an event (such as a symptom) in diseased persons to the probability of that same event in non-diseased persons. LRs indicate how many times more (or less) likely a result is in a patient with the disease compared to a patient free of disease.

The adjectives 'positive' or 'negative' indicate whether the LR refers to the presence of the clinical information (positive) or the absence of the clinical information (negative). Positive LRs with the highest value argue most for disease when the clinical information is present (that is, how the probability changes when the finding is present); negative LRs with the value closest to zero argue the most against disease when that clinical information is absent (that is, how the probability changes when the finding is absent).

Posterior odds: The odds of an event conditional upon another event having occurred.

Prior odds: The odds of an event before acquiring additional information.

Patient presentation (e.g. with symptom) [prior probability]

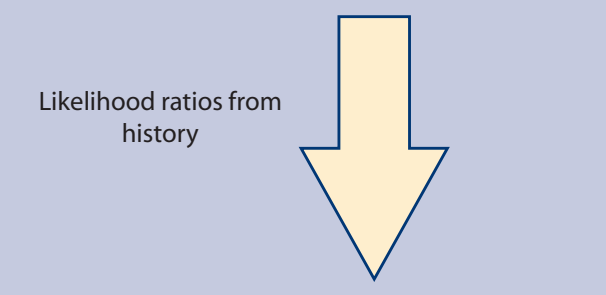

Post-history probability

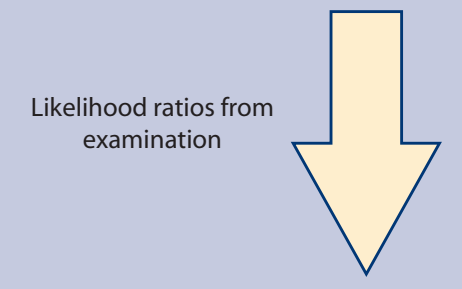

Post-examination probability

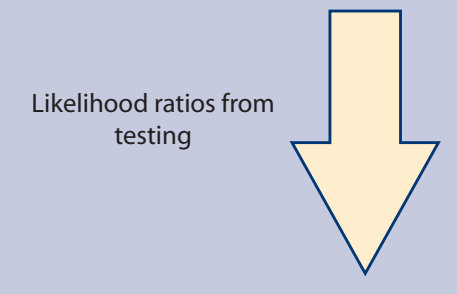

Post-test probability [posterior probability]
Figure 1. The diagnostic processing pathway. 


\section{Table 1. Variation in posterior probability according to prior probability.}

\begin{tabular}{lcccc}
$\begin{array}{l}\text { Prior } \\
\text { probability, \% }\end{array}$ & Prior odds & $\begin{array}{c}\text { Posterior odds } \\
\text { after applying a } \\
\text { LR of 5 }\end{array}$ & $\begin{array}{c}\text { Posterior } \\
\text { probability, \% }\end{array}$ & $\begin{array}{c}\text { Net increase in } \\
\text { probability, \% }\end{array}$ \\
\hline 10 & 0.11 & 0.55 & 35.5 & 25.5 \\
\hline 20 & 0.25 & 1.25 & 55.5 & 35.5 \\
\hline 30 & 0.43 & 2.15 & 68.3 & 38.3 \\
\hline
\end{tabular}

$L R=$ likelihood ratio

\section{Competing interests}

I have authored books entitled Patient-Centred Diagnosis and Diagnosing Cancer in Primary Care. In addition to practising as a GP I also work part-time for NICE as their Consultant Clinical \& Public Health Advisor, but I should like to emphasise that I am writing this piece in my personal capacity as a GP with a longstanding academic interest in diagnostics.

\section{Discuss this article}

Contribute and read comments about this article on the Discussion Forum: http://www.rcgp.org.uk/bjgp-discuss

\section{REFERENCES}

1. Hampton JR, Harrison MJG, Mitchell JRA. Relative contributions of history-taking, physical examination and laboratory investigation to diagnosis and management of medical outpatients. BMJ 1975; 2(5969): 486-489.

(or absence of disease) (Figure 1). ${ }^{6}$

By considering a pathway it becomes clear that in many circumstances, such as the migraine example referred to earlier, the disease probability after the history (the post-history probability) is such that undertaking an investigation may actually be unnecessary whatever the magnitude of its likelihood ratio. Conversely, if diagnostic data is not considered in the context of a diagnostic processing pathway there is an increased risk that investigations may be used inappropriately with a higher chance of false positives in a lower prevalence population.

The importance of a specific mathematical property of likelihood ratios is also more clearly seen in the context of a diagnostic processing pathway. When pre-test probably is plotted against post-test probability according to different levels of likelihood ratios, a series of curves result with the greatest divergence being when the prior probability is greater than 20\%. This suggests that investigations are diagnostically more useful and will have a much greater impact on decision making if the input from the medical history can be maximised (that is, to enhance the magnitude of the post-history probability), (see Table 1 for example calculations). ${ }^{21}$

The medical history is more than a nostalgic relic of little relevance to modern practice. However, there is now an urgent requirement for careful consideration to be given to how, where, and by whom this diagnostic technology is being used. A key component of this initiative would be the development of a library of setting-specific likelihood ratios and $\kappa$ for individual features and combinations of items of medical history information. Subsequently, consideration could be given to extending this to the clinical examination and also to address the requirements of primary care clinicians in a variety of health economies. For example, a recent paper in the Lancet clearly demonstrates the potential public health importance of using simple clinical assessment clusters (incorporating features from both the medical history and the clinical examination) in improving the identification of severe illness in young children within the third world. ${ }^{22}$
2. Speech on the National Health Service. http://www.number10. gov.uk/output/Page14171.asp (accessed 6 Feb 2008).

3. Department of Health. The NHS improvement plan: putting people at the heart of public services. London: The Stationery Office, 2004.

4. National Institute for Health and Clinical Excellence. Guide to the methods of technology appraisal. London: NICE, 2004.

5. Summerton N. Making a diagnosis in primary care: symptoms and context. Br J Gen Pract 2004; 54(505): 570-571. 2006.

7. Department of Health. Huge expansion in NHS's scans from next andstatistics/ pressreleases/DH_4084758 (accessed 6 Feb 2008)

8. No authors listed. Predictors of outcome in headache patients Headache Study Group of The University of Western Ontario. Headache 1986; 26(6): 285-291.

9. Smetana GW. The diagnostic value of historical features in primary headache syndromes: a comprehensive review. Arch Int Med 2000; 160(18): 2729-2737. classification, and psychiatric comorbidity. Arch Intern Med 1993; 153(21): 2474-2480.

11. Summerton N, Mann S, Sutton J, et al. Developing clinically relevant and reproducible symptom-defined populations for cancer diagnostic research in general practice using a community survey. Fam Pract 2003; 20(3): 340-346.

12. Landis JR, Koch GG. The measurement of observer agreement for categorical data. Biometrics 1977; 33(1): 671-679.

13. Sox HC, Hickman DH, Marton KI, et al. Using the patient's history to estimate the probability of coronary artery disease: a comparison of primary care and referral practices. Am J Med 1990; 89(1): 7-14.

14. Bent S, Nallamothu BK, Simel DL. Does this woman have an acute uncomplicated urinary tract infection? JAMA 2002; 287(20): 2701-2710.

15. Metlay JP, Kapoor WN, Fine MJ. Does this patient have communityacquired pneumonia? Diagnosing pneumonia by history and physical examination. JAMA 1997; 278(17): 1440-1445.

16. Whiting P, Toerien M, de Salis I, et al. A review identifies and classifies reasons for ordering diagnostic tests. J Clin Epidemiol 2007; 60(10): 981-989.

17. Smetana GW, Shmerling RH. Does this patient have temporal arteritis? JAMA 2002; 287(1): 92-101.

18. Muris JWM, Starmans R, Fijten $\mathrm{H}$, et al. Non-acute abdominal complaints in general practice: diagnostic value of signs and symptoms. Br J Gen Pract 1995; 45(395): 313-316.

19. Summerton N, Mann S, Rigby AS, et al. Patients with new onset haematuria: assessing the discriminant value of clinical information in relation to urological malignancies. Br J Gen Pract 2002; 52(477): 284-289.

20. Chan SF, Deeks JJ, Macaskill P, Irwig L. Three methods to construct predictive models using logistic regression and likelihood ratios to facilitate adjustment for pretest probability give similar results. J Clin Epidemiol 2008; 61(6): 52-63.

21. McGee S. Evidence-based physical diagnosis. St Louis: Saunders, 2007.

22. The Young Infants Clinical Signs Study Group. Clinical signs that predict severe illness in children under age 2 months: a multicentre study. Lancet 2008; 371(9607): 135-142.
6. Summerton N. Patient-centred diagnosis. Oxford: Radcliffe Publishing, month [press release]. http://www.dh.gov.uk/en/publications presenting to family physicians - a one year prospective study. The

10. Kroenke K, Price RK. Symptoms in the community. Prevalence, 